\title{
Accuracy of CBCT linear
} measurements

\section{Dora Kishkilova}

\author{
1. Department of Diagnostic Imaging and Oral \\ Diagnostic Faculty of Dentistry Medical University \\ Sofia;
}

\begin{abstract}
Accuracy of CBCT measurements has been the subject of many studies. We tried to gather information for the different methods and materials used in these studies and the results. We were particularly interested in researches examining the effect on different factors on the accuracy of measurements. The analysis of data found in literature established that there is no unanimous opinion regarding the combined impact of factors on the accuracy of linear and volumetric measurements using $C B C T$.
\end{abstract}

\section{Keywords: СВCT, accuracy, measurements}

\section{Introduction}

Accuracy of CBCT linear measurements was the subject of many studies and the results are quite debatable. Some authors report mean difference between the radiographic and direct measurements to be very small and insignificant, others state that different factors may affect this accuracy and report differences bigger than $1 \mathrm{~mm}$. СВCT took serious part of imaging in maxillo-facial region and this explains not only the increasing number of studies, evaluating this imaging modality, but also the variety of study designs. In the beginning researches were performed mainly using dry bones and jaws, later cadaver heads were included (human and animal) or simulation of soft tissues were used. 


\section{Results}

Pinsky et al.(1) investigated the accuracy of CBCT measurements using acrylic blocks and human mandibles, in which they created defects with sizes approximating those observed in periodontal diseases or periapical inflammation. The study was carried out in two phases. In the first phase, the authors used acrylic blocks with machined defects of predetermined width and depth. The defects' depth varied from 4 to $10 \mathrm{~mm}$. In the second phase, in order to establish the gold standard, they used the cadaver lower jaws in which they created simulated lesions with two diameters $(2 \mathrm{~mm}$ and $4 \mathrm{~mm})$ and different depths - from 2 to $4.5 \mathrm{~mm}$, with an interval of $0.5 \mathrm{~mm}$. Direct measurements of the defects were carried out using two methods: first linear measurement, and then software-assisted mathematical calculation of the simulated lesions' volume. According to the authors, the method shows high accuracy, which would be affected by the presence of soft tissue and potential movement of the patient during scanning. They recommend a more in-depth analysis of multi-chamber lesions in the jaws, as well as the development of clinical protocols for studying CBCT accuracy. Ahlowalia et al.(2) compared the accuracy of measurements of large jaw defects using CBCT with a limited FOV with those made with MSCT in simulated bone defects created ex vivo in bovine bones, then comparing them with the results from physical measurement of the volumes of the same lesions.

The samples were cut into 20 segments, after which a defect was created in each segment affecting the bone's compactness and spongiosis. According to the authors, this approach creates samples that approximate findings in apical periodontitis, where both compact and cancellous bone can be affected. Defect sizes were selected to correspond to the size and shape of such in apical periodontitis (between 2 and $13 \mathrm{~mm}$ ). Anatomical samples were initially scanned with $40 \mathrm{~mm}$ FOV since, according to the authors, this is the most suitable volume for examining 1 tooth, with 360-degree rotation for $17.5 \mathrm{sec}$. They recommend further studies with a smaller rotation (180 degrees) and shorter duration, with view of decreasing the radiation dose. Two silicone impressions were made of each sample. In terms of physical volumetric measurements, the authors selected two methods. The first method is based on measuring the weight of the silicone imprint and calculating its volume using the information on the material's density provided by the manufacturer. The second method is employing Archimedes' principle according to which any object displaces a volume of water equal to its own volume. The volume of silicone impressions was measured by calculating the difference between the weight of the dry sample and the same placed in water. In the conclusion, the authors state that CBCT is a method entirely equivalent to MSCT for volumetric measurement of simulated bone lesions, as well as for the assessment of the healing process.

Michal Halperin-Sternfeld et al.(3) studied ex vivo accuracy of linear measurements using CBCT by comparing the distances in direct osteometry between defects (reference points) created in advance in porcine mandibles with those obtained on CBCT images. The reference points were spherical cavities created in 6 pre-selected planes, two frontal (to the front of the canines) and four distal (behind the canines), in the lingual and vestibular bone compactness, as well as along the occlusal surface of the tooth in the respective plane. All created defects were filled with radiopaque gutta-percha. After stabilization on polystyrene blocks, the samples were scanned with a CBCT apparatus. Four physical and radiographic measurements were taken for each plane; thickness of the compactness in the immediate vicinity of the reference point contrasted with gutta-percha, vestibular and lingual, the distance between these two points, as well as the distance between the occlusal reference point and the upper border of the mandibular canal. In their results, the authors report that in many cases radiographic measurements are smaller than the physical ones (76\%) - "underestimation" of the radiographic measurement, but report as 
significant the remaining percent (34\%) where measurements on radiographic images are larger than the physical values ("overestimation"), sometimes by $1.09 \mathrm{~mm}$. According to them, these results show an inaccuracy which might be of important clinical significance in potential surgical interventions in the jaws and the overestimation in a radiographic measurement might serve for determining a "safety threshold" in surgical interventions in the maxillofacial area. They put forward two approaches for establishing such a threshold. The first one uses the mean value of overestimation in СBCT measurements, in their case equal to $1.09 \mathrm{~mm}$, since only the overestimation in measurements might lead to damage of important anatomical structures (e.g., mandibular canal). The other approach uses the higher registered value of overestimation in measurements, which in their study is $1.42 \mathrm{~mm}$. The authors recommend the level of the safety threshold to be calculated separately for horizontal and vertical measurements and on the basis of this approach they recommend a "safety zone" of $1.39 \mathrm{~mm}$ and $1.42 \mathrm{~mm}$, respectively.

Mol and Balasundaram et al. (4) compare in vitro accuracy of CBCT images with those of conventional intraoral techniques (retro-alveolar and bite-wing) of human skulls. They place small metal spheres on each of the crown surfaces of available teeth and measure the distance between the enamel-cement order and the top of the interdental septa, relative to these spheres. Results showed better performance of CBCT compared to conventional intraoral techniques, which applied more to the distal jaw sections studied by the authors. The diagnostic accuracy of measurements for the anterior sections was found to be much lower for both methods. The authors attribute this discrepancy to the thinner vestibular and lingual plates in the anterior jaw sections. The inclusion of measurements of lingual and buccal sections of the alveolar bone established CBCT as a method with better diagnostic accuracy compared to intraoral retro-alveolar radiograph due to the fact that $2 \mathrm{D}$ imaging of these sections is more difficult. The authors claim that despite this definite advantage of 3D images, the deviation in measurements in both groups exceeds $1 \mathrm{~mm}$ and might be clinically significant. According to them, such a deviation might be due to subjectivity in the interpretation of radiographic images, resulting from the used voxel size, volumetric and contrast resolution and the signal-to-noise ratio, or a combination of all of the above.

Timock et al.(5) aim in their study to investigate the accuracy and reliability of alveolar bone height and thickness measurements derived from СВСТ images of cadaver human heads, comparing them to direct measurements on the jaws made by dissection. According to the authors, the differences in the measurements derived from CBCT images and direct measurements were close to zero, but when the measurements were divided in two groups - for height and thickness - they had more precise values $0.30 \mathrm{~mm}$ and $0.13 \mathrm{~mm}$, respectively.

Sun et al.(6) investigated how voxel size and bone thickness can influence the accuracy of measurements using CBCT. In their study, they used pig heads, where they created lesions in the jaws projecting under the medial and distal molar tubercles at a distance of at least $4 \mathrm{~mm}$ from the tip of the alveolar ridge. They scanned the samples with two different voxel sizes, $0.2 \mathrm{~mm}$ and $0.4 \mathrm{~mm}$. In the second phase of their study, after reducing bone compactness by $1.5 \mathrm{~mm}$ and $0.5 \mathrm{~mm}$, respectively, the authors aimed in the first case to achieve a bone size smaller than or approximating the voxel size, and in the second - bigger than the voxel size. Scanning was performed with the voxel sizes defined in the first stage. After each scan, they carried out direct measurements of the lesions and linear measurements on the radiographic images. The authors report that the measurements at the pre-reduction phase exhibited overestimation of CBCT measurements, more marked in scanning with voxel size of $0.4 \mathrm{~mm}$. In the post-reduction phase they observe underestimation in CBCT measurements compared to direct measurements, again more marked in scanning with voxel size of $0.4 \mathrm{~mm}$. According to them, although the measurements performed 
with voxel size of $0.2 \mathrm{~mm}$ were closer to measurements with a digital caliper, this was in no way significantly different than those in scanning with voxel size of $0.4 \mathrm{~mm}$.

According to Ryan Wood et al.(7), there is a complex interrelation between the factors affecting the accuracy of measurements using СВCT. According to them, the inaccuracies in bone measurements depend on the voxel size with which scanning is carried out, the presence or absence of soft tissue, the type of software and the localization of the jaw lesions. They claim that from these factors, the only thing that is known is that the voxel size leads to a higher or lower resolution depending on whether the size is increased or decreased, but the interaction between these multiple factors has not been studied yet. In their study, they selected 6 porcine heads of pigs between 3 and 6 months of age. They created grooves in the occlusal surfaces of the molars, as well as round holes in the vestibular, through the gingiva. The holes were 4 in the maxilla and 3 in the mandible. The first scan of the heads used voxel sizes of $0.2 \mathrm{~mm}$ and $0.4 \mathrm{~mm}$, in the presence of soft tissue. In the second phase, they removed the outer soft tissue, cheeks and vestibular gingiva, and kept only the inner ones, tongue and lingual gingiva, and again scanned the heads using the same voxel sizes.

For the physical measurement of the alveolar bone and the two jaws' height and thickness, the authors separated it using a circular saw and additionally cut the samples into more segments, through the middle of the round vestibular holes, the direction of cutting being perpendicular to the occlusal groove created in the teeth. Thus they obtained two mirror surfaces of each hole. Measurements were taken with a digital caliper. Height measurements were taken from the occlusal border of each half of the round hole to the tip of the alveolar ridge, after which the mean value of the measurements in the two mirror halves was calculated. The authors measured the alveolar bone thickness at two pre-selected levels: $0.5 \mathrm{~mm}$ and 1.00 $\mathrm{mm}$ from the tip of the alveolar ridge, perpendicular to the axis of the tooth root, located in the zone of interest, measuring the distance between the vestibular bone surface and lamina corticalis of the respective root.

The authors found a discrepancy between direct measurements in the upper jaw, sometimes reaching 2 $\mathrm{mm}$ and more, and believe that the reason for that lies in the smaller thickness and density of the bone. They claim, contrary to Timock et al., that measurements on images obtained with a voxel size of $0.2 \mathrm{~mm}$ are more accurate only in the presence of all soft tissue (vestibular and lingual). Reducing the voxel size to $0.2 \mathrm{~mm}$ and, respectively, the higher radiation dose is rendered pointless if the soft tissue is removed. They also state that the discrepancy between measurements of the height of the upper jaw alveolar bone on CBCT-derived images with a voxel size of $0.4 \mathrm{~mm}$ and the physical measurements sometimes reaches $2 \mathrm{~mm}$. In the mandible, they report a deviation not exceeding $1 \mathrm{~mm}$.

\section{Conclusion}

Many authors have examined the accuracy of СВCT linear and volumetric measurement the last decade (1-20). The review of literature leads us to the conclusion that the use of CBCT is extremely widespread. Pauwels et al.(21) believe that it is necessary to optimize the radiation dose for the patient, i.e. selection of the most suitable scanning protocol which provides acceptable accuracy for the specific indications. 
The analysis of the data found in research literature established that there is no unanimous opinion regarding the combined impact of factors on the accuracy of linear and volumetric measurements using CBCT.

\section{References}

1. Pinsky HM, Dyda S, Pinsky RW, Misch KA, Sarment DP. Accuracy of three-dimensional measurements using cone-beam CT. Dentomaxillofac Radiol 2006; 35: 410-416

2. Ahlowalia M. S., S. Patel, H. M. S. Anwar, G. Cama, R. S. Austin, R. Wilson \& F. Mannocci Accuracy of CBCT for volumetric measurement of simulated periapical lesions Oral Surgery, Oral Medicine, Oral Pathology, Oral Radiology \& Endodontology. Jan2008, Vol. 105 Issue 1, p91-98. $8 p$.

3. Halperin-Sternfeld M., Machtei E., Horwitz J. Diagnostic Accuracy of Cone Beam Computed Tomography for Dimensional Linear Measurements in the Mandible, The International Journal of Oral \& Maxillofacial Implants Volume 29, Number 3, 2014, p. 593-99

4. Mol and Balasundaram, In vitro cone beam computed tomography imaging of periodontal bone Dentomaxillofacial Radiology (2008) 37, 319-324

5. Timock AM, Cook V, McDonald T, et al. Accuracy and reliability of buccal bone height and thickness measure- ments from cone-beam computed tomography imag- ing. Am J Orthod Dentofacial Orthop 2011; 140:734-744

6. Sun Z, Smith T, Kortam S, Kim DG, Tee BC, Fields H. Effect of bone thickness on alveolar boneheight measurements from cone-beam computed tomography images. Am J Orthod Dentofacial Orthop 2011; 139:e117-27.

7. Wood R, Sun Z, Chaudhry J, Tee BC,Kim DG, LeblebiciogluB,etal. Factors affecting the accuracy of buccal alveolar bone height mea- surements from cone-beam computed tomography images. Am J Orthod Dentofacial Orthop 2013; 143:353- 63

8. Berco M, Rigali PH Jr, Miner RM, DeLuca S, Anderson NK, Will LA. Accuracy and reliability of linear cephalometric measurements from cone-beam computed tomography scans of a dry human skull. Am J Orthod Dentofac Orthop 2009;1 36: 17.e11-19.

9. Brown AA, Scarfe WC, Scheetz JP, Silveira AM, Farman AG (2009) Linear accuracy of cone beam CT derived 3D images. Angle Orthodontist 79, 150-7.

10. Ganguly R, Ruprecht A, Vincent S, Hellstein J, Timmons S, Qian F. Accuracy of linear measurement in the Galileos cone beam computed tomography under simulated clinical conditions. Dentomaxillofac Radiol 2011; 40:299-305.

11. Haiter-Neto F, Wenzel A, Gotfredsen E. Diagnostic accuracy of cone beam computed tomography scans compared with intraoral image modalities for detection of caries lesions. Dentomaxillofac Radiol 2008; 37: 18-22.

12. Lascala CA, Panella J, Marques MM. Analysis of the accuracy of linear measurements obtained by cone beam computed tomography (CBCT-NewTom). Dentomaxillofac Radiol 2004; 33: 291294.

13. Maret D, Telmon N, Peters OA, Lepage B, Treil J, Inglese JM, et al. (2012) Effect of voxel size on the accuracy of 3D reconstructions with cone beam CT. Dentomaxillofac Radiol 41:649-655

14. Marques A.P., Perrella A., Arita E., Pereira E., Cavalcanti M., Assessment of simulated mandibular condyle bone lesions by cone beam computed tomography, Braz Oral Res. 2010 OctDec; 24(4): 467-74

15. Mischkowski RA, Pulsfort R, Ritter L, Neugebauer J, Brochhagen HG, Keeve E, Zoller J E. Geometric accuracy of a newly developed cone-beam device for maxillofacial imaging. Oral Surg Oral Med Oral Pathol Oral Radiol Endod 2007; 104: 551-559

16. Nguyen E, Boychuk D, Orellana M. Accuracy of cone-beam computed tomography in predicting the diameter of unerupted teeth. Am J Orthod Dentofacial Orthop 2011; 140:e59-e66. 
17. Patcas R, Muller L, Ullrich O, Peltomaki T. Accuracy of cone-beam computed tomography at different resolutions assessed on the bony covering of the mandibular anterior teeth. Am J Orthod Den- tofacial Orthop 2012; 141:41-50.

18. Suomalainen A, Vehmas $T$, Kortesniemi M, Robinson S, Peltola J. Accuracy of linear measurements using dental cone beam and conventional multislice computed tomography. Dentomaxillofac Radiol 2008; 37: 10-17.

19. Tomasi C, Bressan E, Corazza B, Mazzoleni S, Stellini E, Lith A (2011) Reliability and reproducibility of linear mandible measure- ments with the use of a cone-beam computed tomography and two object inclinations. Dentomaxillofac Radiol 40:244-250

20. Torres MG, Campos PS, Segundo NP, Navarro M, Crusoe-Rebello I (2012) Accuracy of linear measurements in cone beam computed tomography with different voxel sizes. Implant Dent 21:150-155

21. Pauwels R, Beinsberger J, Collaert B, Theodorakou C, Rogers J, Walker A, et al. The SEDENTEXCT Project Consortium. Effective dose range for dental cone beam computed tomography scanners. Eur J Radiol 2012; 81: 267-271.

\section{Corresponding author:}

Dora Kishkilova,

Department of Diagnostic Imaging

and Oral Diagnostic,

Faculty of Dentistry,

Medical University - Sofia,

1.Georgi Sofijski Blvd,

Sofia, Bulgaria

Tel.: +359 887777807 